\title{
Stigma as a barrier to diagnosis of lung cancer: patient and general practitioner perspectives
}

\author{
Nicola Scott ${ }^{1,4}$, Melanie Crane ${ }^{2}$, Mayanne Lafontaine ${ }^{3}$, Holly Seale ${ }^{4}$ and David Currow ${ }^{3}$ \\ ${ }^{1}$ NSW Public Health Officer Training Program, NSW Ministry of Health, Sydney, Australia \\ ${ }^{2}$ School of Public Health, University of Sydney, NSW, Australia \\ ${ }^{3}$ Cancer Institute NSW, Eveleigh, Sydney, Australia \\ ${ }^{4}$ School of Public Health and Community Medicine, University of New South Wales, Sydney, Australia
}

\begin{abstract}
The prognosis for people with lung cancer may be worsened by delays in seeking medical help following the onset of symptoms. Previous research has highlighted that patients' experiences of stigma and blame may contribute to these delays. This short report focuses on stigma as a barrier to diagnosis of lung cancer, from patient and general practitioner (GP) perspectives. Semi-structured interviews were conducted with people diagnosed with lung cancer $(n=20)$ and with GPs $(n=10)$ in New South Wales, Australia. Participants' experiences of blame and stigma, GPs preconceptions of lung cancer risk and the impact of anti-smoking messaging were explored. Participants reported experiencing stigma owing to a diagnosis of lung cancer. For some, the anticipation of stigma resulted in delays in seeking diagnosis and hence treatment. The sense of blame associated with a lung cancer diagnosis was also reflected in GP interviews. Successful tobacco control activities have increased societal awareness of lung cancer as smoking related and potentially contributed to the participants' experiences of stigma. Removing blame associated with smoking is central to reducing delays in diagnosis of lung cancer.
\end{abstract}

Key words: general practitioners; lung cancer; smoking; stigma

Received 7 December 2013; revised 26 November 2014; accepted 9 January 2015; first published online 9 February 2015

\section{Introduction}

Stigma is a multifaceted social process that can be anticipated or experienced (Lebel and Devins, 2008). Definitions of stigma within social psychology agree that it consists of an attribute that marks people as different, leading to a perceived devaluation (Yang et al., 2007). These definitions also acknowledge that stigma is socially constructed, dependent on both relationship and context (Yang et al., 2007). Stigma has been further described as 'exclusion, rejection, blame or devaluation in response to features such as physical deformities, undesirable personal characteristics or membership in a despised social group' (Lebel and Devins, 2008: 730).

Correspondence to: Nicola Scott, Cancer Institute, NSW, P.O. Box 41, Alexandria, NSW 1435, Australia. Email: nicola. scott@cancerinstitute.org.au
Stigma has been linked to negative outcomes across a range of areas in health. Stigma in cancer can be exacerbated when people's voluntary behaviour is believed to have contributed to disease onset (Lebel and Devins, 2008). In relation to lung cancer, stigma may occur as a result of the association between the disease and smoking, the perception of the disease as self-inflicted; its high mortality; and perceptions about the type of death that may be experienced (Chapple et al., 2004). Conversely, smoking-related stigma can also have positive public health benefits such as dissuading people to take up smoking and influencing people to quit smoking (Stuber et al., 2008).

Lung cancer is often diagnosed in the later stages of the disease and time between symptom onset and consultation with a doctor can be long enough plausibly to affect prognosis (Chatwin and Sanders, 2013). Detection of lung cancer in earlier stages generates better outcomes and improves

(C) Cambridge University Press 2015 
survival rates across the population (McWilliams et al., 2009). A number of barriers can delay the detection of lung cancer including fear of health professionals' censure associated with smoking (Tod and Joanne, 2010). Evidence suggests that a longer history of smoking across the population contributes to greater time delays in seeking medical help, and this may in part be because of the fear of blame and stigma attached to smoking (Chapple et al., 2004; Smith et al., 2009).

The aim of this short report is to explore the attitudes and beliefs of people with lung cancer and general practitioners (GP) in relation to stigma as a barrier to diagnosis of lung cancer. It is part of a larger study that focuses on lung cancer awareness, knowledge and beliefs across New South Wales (NSW), Australia.

\section{Methods}

Semi-structured in-depth interviews were conducted with people who had been diagnosed with lung cancer and with GPs in NSW, Australia. Participants were recruited for the study through lung cancer support networks, across metropolitan and regional areas. To understand experiences in relation to their diagnosis, interviews of people with lung cancer explored personal experiences and investigated participant's recall of symptoms and behaviours that prompted them to seek medical attention. Interviews with GPs explored the practitioners' perceptions and understanding of lung cancer signs and symptoms, current practices and barriers to referral.

Questions were asked in an open-ended manner to allow room for expansion; prompts were only given when the interviewer deemed that it was necessary to encourage conversation back to topic or to address a certain issue. Interviews were facilitated by experienced qualitative researchers. Ethics clearance was obtained from the NSW Population \& Health Services Research Ethics Committee and all participants gave written informed consent. The consolidated criteria for reporting qualitative research checklist for qualitative research was used to guide analysis and reporting (Tong et al., 2007). The interviews were digitally recorded, transcribed verbatim and analysed thematically using QSR International's NVIVO10 software.

\section{Results}

\section{Participants}

All of the participants $(n=20)$ with lung cancer were over 40 years of age and were former smokers $(n=13)$ or non-smokers $(n=7)$. None of the participants were current smokers at the time of the research. Interviews were conducted with male $(n=6)$ and female $(n=4)$ GPs, from metropolitan or regional NSW $(n=10)$.

\section{Experiences of blame and stigma}

Most participant's reported feeling stigmatised, with some also experiencing blame from health professionals and the general community. A number of smokers reported that they postponed a consultation with their GP, as they felt more comfortable approaching their GP as a former, rather than a current smoker. In one case, this led to significant delays in seeking treatment for symptoms.

'I thought, "I can't go and see [DOCTOR] while I still smoke" - I just had this notion that I'd really feel kind of silly going to him saying, "Look, I don't feel well. I smoke at least 10 cigarettes a day. Fix me up." And I felt kind of stupid doing that so I thought, "I'll give up smoking, then I'll go and see [my doctor]”'.

(Male, former smoker)

Reactions from the general community to the news of a diagnosis of lung cancer highlighted the far reaching nature of smoking-related stigma.

'Because the moment you tell somebody you've got lung cancer, the first thing out of their mouth is, "Well, when did you stop smoking? How long did you smoke for?" It's not, "My God! That is just so awful. How are you doing?",

(Female, non-smoker)

Participants also contrasted the stigma surrounding lung cancer with other cancers, such as breast cancer that attracts more attention and research funding than lung cancer. Participants also expressed concern that survival rates for lung cancer are unlikely to improve until the blame associated with the disease is reduced. They felt lung cancer treatments are unlikely to attract interest and funding while there continues to be a 
perception that lung cancer is the 'fault' of the individual.

'People see me with a bald head and ask "have you got breast cancer?" "No, I have lung" "Oh, well, you're a smoker"'.

(Female, former smoker)

\section{GPs preconceptions of lung cancer risk}

During the GP interviews, there was a sense of blame with regards to a diagnosis of lung cancer, because it was typically seen as having been caused by someone's choice to smoke for many years.

'You have the most sympathy for those who do get it and they've never smoked a cigarette and it's just unfair'.

(GP metropolitan area)

'Because it presents so late, there's not much you can really do except to tell people "If you get it because you were smoking, well we told you, you shouldn't have smoked. If you didn't smoke at all and you still got it, you're just really unlucky"'.

$$
\text { (GP metropolitan area) }
$$

GPs also agreed that from their experience lung cancer patients often delay presentation following symptom onset.

Delays in diagnosis were also suggested to occur when GPs do not consider that a non-smoker may be at risk of lung cancer and as a result rule out lung cancer and pursue treatments for other conditions.

'So, that's also why there's a delay because you'll say, "Oh it could be this it could be that" and then you work through your other differential diagnoses to get there'.

(GP regional area)

Some participants with lung cancer reported that they felt their GP did explore all options, irrespective of their smoking status.

'I know with my GP that never came into the conversation. It was, "You've got a symptom there that we need to find out what's causing it ...”. But never did he say, "Oh, you shouldn't have to worry because you've never smoked"'.

(Female, non-smoker)

\section{Anti-smoking messaging}

Participants highlighted that anti-smoking messaging portrays lung cancer as a fatal disease and potentially facilitates the stigma that is felt by lung cancer patients - irrespective of their smoking status.

'I think there's such a stigma attached to lung cancer. More work needs to be done to take that - I mean, I get what the government's trying to do with its advertising to stop people from smoking, but what they're actually doing is harming every single lung cancer patient that is fighting this disease'.

(Female, non-smoker)

Views of lung cancer as a 'death sentence' and severe health consequence of smoking (as portrayed in some anti-smoking messaging) meant that participants were hesitant to seek medical advice for symptoms that had the potential to be lung cancer, causing delays in diagnosis. Some GPs interviewed also felt that the anti-smoking TV advertising raised fear levels in smokers to such an extent that it may deter them from seeking medical advice.

Owing to societal knowledge of the association between smoking and lung cancer, participants who were non-smokers felt the need to clarify their smoking status to those around them including clinicians, to minimise blame.

'[The TV ads are] all about, "Look what's going to happen if you smoke" and therefore there is this inextricable link between smoking and lung cancer which perpetuates this problem both in the doctors and in the general community, ... Mum, whenever she would tell anyone that I had lung cancer, it would be immediately followed by "but he never smoked". Because of that stigma, like you've brought it on or you're partly to blame or you've contributed'.

(Male, non-smoker)

\section{Discussion}

International research suggests that stigma related to lung cancer is commonly experienced (Chapple et al., 2004; Tod et al., 2007; Raleigh, 2010). A systematic review on the impact of stigma and nihilism on lung cancer outcomes concluded that 
health-related stigma is part of the lung cancer experience, contributing to psychological distress for patients and impairing quality of life (Chambers et al., 2012). In one UK study, stigma was a prevalent factor leading to delays in reporting symptoms (Tod et al., 2007). Some nonsmokers or ex-smokers delayed reporting symptoms because of an expectation, that they would be stigmatised as a smoker and blamed for their illness (Tod et al., 2007). Stigma appears to have a tangible influence on the point at which some people decide to seek medical help following symptom onset (Chatwin and Sanders, 2013).

The influence of stigma on health professionals is unclear; however, deficits were found in health professionals' knowledge of contemporary evidencebased lung cancer care (Chambers et al., 2012). For healthcare professionals, care and sensitivity is needed when treating patients with illnesses that are perceived to be potentially 'self-inflicted'. Many older adults had started smoking at a time when smoking was socially acceptable and when tobacco was even provided free during national service (Chapple et al., 2004). Interventions to reduce stigma amongst the medical community are needed, with the aim of minimising delays in diagnosis of lung cancer.

Successful tobacco control activities such as mass media campaigns have raised social awareness of the health consequences of smoking, including lung cancer. These activities have had a positive influence on reducing smoking rates and uptake (Wakefield et al., 2008); however, they may have also contributed to creating a negative impact on those with lung cancer (Chambers et al., 2012). This is a complex matter, and a multi-level approach may be required to reduce the stigma associated with lung cancer. These approaches include: (1) anti-smoking messages to prevent and reduce the incidence of smoking-related lung cancer, (2) awareness raising of the symptoms consistent with lung cancer to increase earlier detection of lung cancer amongst smokers, former smokers and never smokers and (3) social or community education activities to combat health-related stigma in lung cancer (Chambers et al., 2012).

Limitations of the study include: (1) checking of themes was not undertaken by participants, (2) a single coder was used, (3) interviews were only undertaken with a select group of participants, so the possibility of other important themes emerging cannot be ruled out. Gaining perspectives on this topic from other health professionals such as cancer specialists, both medical and nursing would also be beneficial. This was a qualitative study, and key findings should be explored further in larger, quantitative studies.

\section{Conclusion}

The collective experiences of participants in this study has highlighted the stigma in the community and amongst GPs towards people diagnosed with lung cancer, particularly in relation to smoking. Participants in this research felt that disclosing a diagnosis of lung cancer meant that people assumed that they smoked, and carrying an implication that the individual was responsible for developing their lung cancer.

GP's may be required to balance anti-smoking messages with encouraging patients to seek help when experiencing smoking-related symptoms. Removing the blame associated with lung cancer could be an important factor in reducing delays in the diagnosis of lung cancer. For smokers, stigma can serve to delay seeking treatment following symptom onset, as highlighted by participants in this research waiting until they quit smoking to approach their GPs. For never smokers, the link between smoking and lung cancer means that they (and their GPs) are less likely to perceive themselves as being at risk. While acknowledging the link to smoking status, it may be important to focus attention on symptoms themselves as a way of prompting earlier detection of lung cancer.

\section{Acknowledgements}

The authors would like to thank the participants of this study and Jenny and John Crawford from Curly Questions Pty Ltd for conducting the interviews.

\section{Financial Support}

This analysis was completed while Nicola Scott was an employee of the NSW Public Health Officer Training Program, funded by the NSW Ministry of Health, while based at the Cancer Institute NSW. Dr Holly Seale is supported by an NHMRC

Primary Health Care Research \& Development 2015; 16: 618-622 
Australian-based Public Health Training Fellowship (1012631).

\section{Conflicts of Interest}

None.

\section{Ethical Standards}

Ethics approval for this study was gained from the New South Wales Population \& Health Services Research Ethics Committee (AU RED Reference: HREC/12/CIPHS/20 and LNR/12/CIPHS/29).

\section{References}

Chambers, S.K., Dunn, J., Occhipinti, S., Hughes, S., Baade, P., Sinclair, S., Aitken, J., Youl, P. and O'connell, D.L. 2012: A systematic review of the impact of stigma and nihilism on lung cancer outcomes. BMC Cancer 12, 184.

Chapple, A., Ziebland, S. and Mcpherson, A. 2004: Stigma, shame and blame experienced by patients with lung cancer: qualitative study. British Medical Journal 328, 1470.

Chatwin, J. and Sanders, C. 2013: The influence of social factors on help-seeking for people with lung cancer. European Journal of Cancer Care 22, 709-13.

Lebel, S. and Devins, G.M. 2008: Stigma in cancer patients whose behaviour may have contributed to their disease. Future Oncology 4, 717-33.
Mcwilliams, A., Lam, B. and Sutedja, T. 2009: Early proximal lung cancer diagnosis and treatment. European Respiratory Journal 33, 656-65.

Raleigh, Z.T. 2010: A biopsychosocial perspective on the experience of lung cancer. Journal of Psychosocial Oncology 28, 116-25.

Smith, S.M., Campbell, N.C., Lee, A.J., Raja, A., Wyke, S., Ziebland, S.B., Duff, E.M., Ritchie, L.D. and Nicolson, M.C. 2009: Factors contributing to the time taken to consult with symptoms of lung cancer: a cross-sectional study. Thorax 64 , 523-31.

Stuber, J., Galea, S. and Link, B.G. 2008: Smoking and the emergence of a stigmatized social status. Social Science \& Medicine 67, 420-30.

Tod, A.M., Craven, J. and Allmark, P. 2007: Diagnostic delay in lung cancer: a qualitative study. Journal of Advanced Nursing 61, 336-43.

Tod, A.M. and Joanne, R. 2010: Overcoming delay in the diagnosis of lung cancer: a qualitaitve study. Nursing Standard 24, 35-43.

Tong, A., Sainsbury, P. and Craig, J. 2007: Consolidated criteria for reporting qualitative research (COREQ): a 32-item checklist for interviews and focus groups. International Journal for Quality in Health Care 19, 349-57.

Wakefield, M.A., Durkin, S., Spittal, M.J., Siahpush, M., Scollo, M., Simpson, J.A., Chapman, S., White, V. and Hill, D. 2008: Impact of tobacco control policies and mass media campaigns on monthly adult smoking. American Journal of Public Health 98, 1443-450.

Yang, L.H., Kleinman, A., Link, B.G., Phelan, J.C., Lee, S., Good, B. 2007: Culture and stigma: adding moral experience to stigma theory. Social Science \& Medicine 64, 1524-535. 